\title{
GENERALIZED STATISTICS AND DYNAMICS IN CURVED SPACETIME
}

\author{
V.Bardek, S.Meljanac and A.Perica \\ Rudjer Bošković Institute, P.O.B. 1016, \\ 41001 Zagreb, Croatia
}

(January 4, 1994)

\begin{abstract}
We consider the generalized momentum-depending quon algebra in a dynamically evolving curved spacetime and perform a type of analysis similar to that of J.W.Goodison and D.J.Toms. We find that, at least in principle, all kinds of statistics may occur in some regions, i.e. phases in momentum space, depending on Bogoliubov coefficients determined by a specific dynamical model.
\end{abstract}

PACS numbers: 05.30. $-d, 04.60 .+n$ 
The problem of allowed statistics, such as Bose, Fermi, para-Bose, para-Fermi, anyonic, infinite-quonic, and their role in physics is of great importance [1-4]. It has recently been claimed [5] that, starting with the global quon algebra [2] no generalized statistics is compatible with the dynamics in curved spacetime. The only allowed possibilities found are the ordinary Bose and Fermi statistics. However, the initial assumption that the parameter of deformation $q$ is a real constant is too restrictive. Namely, two of us [6] proposed a more general quon algebra interpolating between all types of statistics simultaneously, and having the same basic properties as the global quon algebra [2].

In this Letter, starting with the generalized quon algebra, we classify and discuss possible statistics compatible with gravitationally induced particle creation in a dynamically evolving universe.

The generalized quon algebra [6] is given by

$$
a_{i} a_{j}^{\dagger}-q_{i j} a_{j}^{\dagger} a_{i}=\delta_{i j}, \forall i, j \in S
$$

Here $q_{i j}=q_{j i}^{*}$ are complex functions and $\mathrm{S}$ is a set of (discrete or continuos) 3-dimensional momenta. Norms in the Fock space are positive definite if $\left|q_{i j}\right| \leq 1$, for all pairs $i, j \in S$. If $\left|q_{i j}\right|<1$, there are no commutation relations between $a_{i}, a_{j}$ (or $a_{i}^{\dagger}, a_{j}^{\dagger}$ ) operators. However, in the exact limit when $\left|q_{i j}\right|=1$ for every pair of indices $i, j \in S$, the following commutation relations are valid:

$$
a_{i} a_{j}-q_{i j}^{*} a_{j} a_{i}=0, \forall i, j \in S
$$


In this case the number operator is $N_{i}=a_{i}^{\dagger} a_{i}$.

We consider generalized quon statistics in a dynamically evolving curved spacetime which, prior to some initial time $t_{1}$ and subsequent to some later time $t_{2}$, is flat.The spacetime is dynamic for $t_{1} \leq t \leq t_{2}$ and given by the spatially flat Robertson-Walker metric [7]. In the first flat region where $t<t_{1}$ (in-region) we assume that annihilation and creation operators $a_{i}, a_{i}^{\dagger}$ satisfy the generalized quon algebra, Eq.(1).In the second flat region where $t>t_{2}$ (out-region) we assume that the corresponding annihilation and creation operators $b_{i}, b_{i}^{\dagger}$ satisfy the generalized quon algebra:

$$
b_{i} b_{j}^{\dagger}-q_{i j}^{\prime} b_{j}^{\dagger} b_{i}=\delta_{i j}, \forall i, j \in S .
$$

We have denoted these operators by $b_{i}$ and $b_{i}^{\dagger}$ because they, in general, differ from the corresponding one in the in-region. The general relation between the two sets of in- and out- operators is given by

$$
a_{i}=\sum_{j}\left(\alpha_{j i} b_{j}+\beta_{j i}^{*} b_{j}^{\dagger}\right),
$$

where $\alpha_{j i}, \beta_{j i}$ are Bogoliubov coefficients for the expanding universe [5], [7]. However, for the spacetime with the Robertson-Walker metric between the in- and outregions, $t_{1}<t<t_{2}$, the Bogoliubov coefficients are diagonal [7]. Therefore, we restrict ourselves to the case where 


$$
\begin{aligned}
& \alpha_{i j}=\alpha_{i} \delta_{i j}, \\
& \beta_{i j}=\beta_{i} \delta_{i j} .
\end{aligned}
$$

Substituting $(4,5)$ and its complex conjugate into (1), we easily obtain

$$
\begin{aligned}
& \alpha_{i} \alpha_{j}^{*}\left(b_{i} b_{j}^{\dagger}-q_{i j} b_{j}^{\dagger} b_{i}\right)+\beta_{i}^{*} \alpha_{j}^{*}\left(b_{i}^{\dagger} b_{j}^{\dagger}-q_{i j} b_{j}^{\dagger} b_{i}^{\dagger}\right)+\alpha_{i} \beta_{j}\left(b_{i} b_{j}-q_{i j} b_{j} b_{i}\right) \\
& +\beta_{i}^{*} \beta_{j}\left(b_{i}^{\dagger} b_{j}-q_{i j} b_{j} b_{i}^{\dagger}\right)=\delta_{i j} .
\end{aligned}
$$

Taking the expectation value of (6) with a vacuum state in the out-region yields,

$$
\left|\alpha_{i}\right|^{2}-q_{i i}\left|\beta_{i}\right|^{2}=1, \forall i \in S
$$

From (7) it follows that (6) can be reduced to

$$
\begin{aligned}
& \alpha_{i} \beta_{j}\left(b_{i} b_{j}-q_{i j} b_{j} b_{i}\right)+\beta_{i}^{*} \alpha_{j}^{*}\left(b_{i}^{\dagger} b_{j}^{\dagger}-q_{i j} b_{j}^{\dagger} b_{i}^{\dagger}\right)+\left(1-q_{i j} q_{j i}^{\prime}\right) \beta_{i}^{*} \beta_{j} b_{i}^{\dagger} b_{j}+ \\
& \left(q_{i j}^{\prime}-q_{i j}\right) \alpha_{i} \alpha_{j}^{*} b_{j}^{\dagger} b_{i}=0 .
\end{aligned}
$$

It is easy to see that the remaining relations following from (8) are

$$
\begin{gathered}
\alpha_{i} \beta_{j}\left(b_{i} b_{j}-q_{i j} b_{j} b_{i}\right)=0, \forall i, j \in S \\
\beta_{i}^{*} \alpha_{j}^{*}\left(b_{i}^{\dagger} b_{j}^{\dagger}-q_{i j} b_{j}^{\dagger} b_{i}^{\dagger}\right)=0, \forall i, j \in S \\
\left(1-q_{i j} q_{i j}^{*}\right) \beta_{i}^{*} \beta_{j}=0, i \neq j \\
\left(q_{i j}^{\prime}-q_{i j}\right) \alpha_{i} \alpha_{j}^{*}=0, i \neq j \\
\left(q_{i i}-q_{i i}^{\prime}\right)\left|\alpha_{i}\right|^{2}=\left(1-q_{i i} q_{i i}^{\prime}\right)\left|\beta_{i}\right|^{2}, \forall i \in S .
\end{gathered}
$$

Now, according to the possible values of Bogoliubov coefficients, we shall classify and discuss all possible solutions of the conditions (7), (9). 
Solution (i): $\alpha_{i} \beta_{i} \neq 0, \forall i \in S$

From the first of Eqs. (9) it follows that

$$
b_{i} b_{j}-q_{i j} b_{j} b_{i}=0, \forall i, j \in S
$$

and its hermitian conjugate. These commutation relations between $b_{i}, b_{j}$ can exist only if $\left|q_{i j}\right|=1$ for $\forall i, j \in S$, [6].

From Eq. (2) it follows that $q_{i j}^{*}=q_{i j}$. Hence, $q_{i j}^{2}=1$, i.e., $q_{i j}= \pm 1, \forall i, j \in S$. Furthermore, from the fourth and fifth of Eqs. (9) one obtains $q_{i j}^{\prime}=q_{i j}$. Let us summarize solution (i): $q_{i j}^{\prime}=q_{i j}, q_{i j}^{2}=1$ for every pair i,j.

Let us further discuss two possible cases. First, if the index $i$ is a continuous variable, then the functions $\alpha_{i}, \beta_{i}$ are continuous functions of i. It follows from Eq. (7) that $q_{i i}=$ const., i.e., $q_{i i}=+1, \forall i \in S$ or $q_{i i}=-1, \forall i \in S$. There are no additional restrictions on $q_{i j}$ if $i \neq j$. Note that, in the above considerations, $q_{i j}$ has not necessarily been a continuous function. (However, if we assume that $q_{i j}$ is also a continuous function in the variables $\mathrm{i}, \mathrm{j}$ then $q_{i j}=$ const. $=+1$ or $-1, i, j \in S$.) Note that $q_{i i}$ would have jumps if $\beta_{i}$ could vanish.

The second possibility assumes that in addition to the continuous variable $i$ there is a discrete variable $\mu=1, \ldots ., p$. All the conditions (Eqs.(7), (9)) remain the same under the following substitutions: $\alpha_{i}, \beta_{i} \rightarrow \alpha_{i}^{\mu}, \beta_{i}^{\mu}, a_{i}, b_{i} \rightarrow a_{i}^{\mu}, b_{i}^{\mu}$, and $q_{i j} \rightarrow q_{i \mu, j \nu}$. If we assume that $q_{i \mu, j \nu}$ is a continuous function in the $\mathrm{i}, \mathrm{j}$ variables, then $q_{i \mu, j \nu}$ reduces to $q_{\mu \nu}$. Solution (i) reads: $q_{\mu \mu} \neq$ const., $q_{\mu \nu}^{\prime}=q_{\mu \nu}, q_{\mu \nu}^{2}=1, \mu, \nu=1, \ldots, p$.

Let us write the corresponding algebra: 


$$
\begin{gathered}
a_{i}^{\mu} a_{j}^{\nu \dagger}-q_{\mu \nu} a_{j}^{\nu \dagger} a_{i}^{\mu}=\delta_{i j} \delta_{\mu \nu}, \forall \mu, \nu=1, \ldots, p \\
a_{i}^{\mu} a_{j}^{\nu}-q_{\mu \nu} a_{j}^{\nu} a_{i}^{\mu}=0, \forall i, j \in S .
\end{gathered}
$$

This is a generalization of Green's oscillator algebra [四. In the special case $q_{\alpha \beta}= \pm\left(2 \delta_{\alpha \beta}-1\right)$, one obtains p Green's oscillators of the Bose (Fermi) type for the upper (lower) sign. Hence, para-Bose and para-Fermi statistics are also allowed, in addition to the ordinary Bose and Fermi statistics.

Solution (ii): $\alpha_{i} \neq 0, \beta_{i}=0, \forall i \in S$

Equation (7) gives $\left|\alpha_{i}\right|=1, i \in S$ and the fourth and the fifth of Eqs. (9) give $q_{i j}^{\prime}=q_{i j}$. In this case there are no restrictions on statistics. We can have arbitrary $q_{i j}$, with $\left|q_{i j}\right| \leq 1$ and $q_{i j}^{\prime}=q_{i j}$. In this case anyons in a multivalued picture [8] are also allowed.

Solution (iii): $\alpha_{i}=0, \beta_{i} \neq 0, \forall i \in S$

From Eq. (7) it follows that $-q_{i i}\left|\beta_{i}\right|^{2}=1$ and $q_{i i}<0, \forall i \in S$. Since $\beta_{i} \beta j^{*} \neq 0$, the third of Eqs. (9) gives $q_{i j}^{\prime *}=q_{i j}^{-1}$. In addition, $\left|q_{i j}^{\prime}\right| \leq 1$ and $\left|q_{i j}\right| \leq 1$, therefore $\left|q_{i j}^{\prime}\right|=\left|q_{i j}\right|=1$. Hence, we find that $q_{i j}^{\prime}=q_{i j}=e^{i \triangle_{i j}}, i \neq j$, where $\triangle_{i j}=-\triangle_{j i}$ is a real function. For $i=j, q_{i i}^{\prime}=q_{i i}^{-1}$, and in the same way as above we find that $q_{i i}^{\prime}=q_{i i}= \pm 1$. Since $q_{i i}<0$, the only possibility is $q_{i i}^{\prime}=q_{i i}=-1$, which means the hard-core condition for all oscillators: $a_{i}^{2}=b_{i}^{2}=0, \forall i \in S$.

Let us summarize solution (iii):

$q_{i j}^{\prime}=q_{i j}=e^{i \triangle_{i j}}, i \neq j$ and $q_{i i}^{\prime}=q_{i i}=-1, \forall i \in S$. It corresponds to anyons with the hard-core condition in a single-valued picture [9]. 
General solution (iv):

Solutions (i), (ii) and (iii) are characterized by the respective restriction s: $\alpha_{i} \beta_{i} \neq$ $0, \forall i \in S ; \alpha_{i} \neq 0, \beta_{i}=0, \forall i \in S ; \alpha_{i}=0, \beta_{i} \neq 0, \forall i \in S$. According to [5], [0], solution (ii) is not physically acceptable. In a similar way, solution (iii) seems to be not allowed unless there is a special dynamical reason for that. So we are left with solution (i) allowing the Bose, Fermi, and generalized Green's oscillator algebra to be consistent with the dynamical evolution of spacetime.

However, even solution (i), might not be realistic. Therefore, we now assume no restrictions on $\alpha_{i}, \beta_{i}$, except the necessary condition Eq.(7). Let us decompose the initial set of indices i into three not overlapping subsets:

$$
\begin{gathered}
M_{1}=\left\{i \in S, \alpha_{i} \beta_{i} \neq 0\right\}, \\
M_{2}=\left\{i \in S, \beta_{i}=0\right\}, \\
M_{3}=\left\{i \in S, \alpha_{i}=0\right\} .
\end{gathered}
$$

Note that $M_{1} \cup M_{2} \cup M 3=S$.

It is obvious that solution (1) applies to set $M_{1}$, solution (ii) to set $M_{2}$, and solution (iii) to set $M_{3}$. Equations (9) with indices i,j from different subsets , $\left(M_{1}, M_{2}\right)$, $\left(M_{1}, M_{3}\right),\left(M_{2}, M_{3}\right)$, are satisfied by $q_{i j}^{\prime}=q_{i j}= \pm 1$. Hence the general solution for $\alpha_{i}, \beta_{i}$, calculated in a dynamical model of evolving spacetime, allows all types of statistics, at least in principle. The main conclusion is that $q_{i j}^{\prime}=q_{i j}$, i.e., that $q_{i j}$ cannot be changed in the course of dynamical evolution. The question of existence, structure, and topology of subsets $M_{2}, M_{3}$ is deeply connected with a concrete dynamical model of evolving spacetime, and is beyond the scope of this paper.

Nevertheless, let us assume that nontrivial sets $M_{2}, M_{3}$ exist. Hence, there may 
exist several different regions (i.e., "phases" in momentum space) with solutions $M_{1}$ of the Bose or Fermi type. The regions are separated by a boundary of the type $M_{2}$ or $M_{3}$. All types of statistics (including infinite-quonic statistics) are possible on the $M_{2}^{\beta=0}$ boundary. Only anyons with the hard-core condition are possible on the $M_{3}^{\beta=0}$ boundary. Transition from one $M_{1}$ phase to another $M_{1}$ phase through $M_{3}^{\beta=0}$ may change the Bose-type to Fermi-type of statistics. However, transition through $M_{3}^{\alpha=0}$ is possible only if the neighboring phases are of the Fermi-type and, hence, cannot change the type of statistics.

Finally, let us mention that our analysis, although general, is still restricted to the generalized quon algebra (Eq.(1)). However, there are other deformed algebras [10], not included in Eq.(1), for which a similar analysis can be performed.

This work was supported by the Scientific Fund of the Republic of Croatia. 


\section{References}

[1] H.S.Green, Phys.Rev.90, 270 (1953).

Y.Ohnuki and S.Kamefuchi, Quantum Field Theory and Parastatistics (University of Tokyo Press, Tokyo, Springer, Berlin, 1982).

[2] O.W.Greenberg, Phys.Rev.Lett.64, 705 (1990), Phys.Rev.D43, 4111 (1991).

[3] O.W.Greenberg, D.M.Greenberger, and T.V.Greenbergest, in Proceedings of the International Conference on Fundamental Aspects of Quantum Theory, 1993.

A.Strominger, Phys.Rev.Lett.71, 3397 (1993).

[4] J.M.Leinaas and J.Myrheim, Nuovo Cim.37B, 1 (1977). F.Wilczek, Phys.Rev.Lett.48, 114 (1982).

[5] J.W.Goodison and D.J.Toms, Phys.Rev.Lett.71, 3240 (1993).

[6] S.Meljanac and A.Perica, Bose, Fermi, para-Bose, para-Fermi, and anyon statistics: The R-matrix approach to deformed associative algebras., submitted to Phys.Rev.Lett.

[7] L.Parker, Phys.Rev.183, 1057 (1969), Phys.Rev.D3, 346 (1971).

N.D.Birrell and P.C.W.Davies, Quantum fields in curved space (Cambridge University Press, 1982).

[8] V.Bardek, M.Doresic, and S.Meljanac, Anyons as quon particles, to be published in Phys.Rev.D. 
[9] A.Lerda and S.Sciuto, Nucl.Phys.B401, 613 (1993).

[10] W.Pusz and S.Woronowicz, Rep.Math.Phys.27, 231 (1989). D.B.Fairlie and C.K.Zachos, Phys.Lett.B256, 43 (1991). 